\title{
Enema versus polyethylene glycol for the management of rectal faecal impaction in children with constipation - a systematic review of randomised controlled trials
}

\author{
Piotr Dziechciarz, Katarzyna Wojtyniak, Andrea Horvath, Hanna Szajewska \\ Department of Paediatrics, Medical University of Warsaw, Warsaw, Poland
}

Prz Gastroenterol 2015; 10 (4): 234-238

DOI: 10.5114/pg.2015.52184

Key words: rectal faecal impaction, functional constipation, polyethylene glycol, enema.

Address for correspondence: Piotr Dziechciarz MD, Department of Paediatrics, Medical University of Warsaw, 1 Dzialdowska St, 01-184 Warsaw, Poland, phone/fax: +48 2245233 09, e-mail: piotrdz@hotmail.com

\begin{abstract}
Introduction: Rectal faecal impaction (RFI) from functional constipation is a common problem in children. Maintenance therapy should start after successful disimpaction. However, there is uncertainty with regard to the optimal disimpaction regimen. Aim: We systematically evaluated the effect of polyethylene glycol (PEG) compared to enema for treating RFI.

Material and methods: The MEDLINE, EMBASE, and the Cochrane Library, with no language restrictions, were searched up to July 2014 for randomised controlled trials (RCTs) evaluating the effect of PEG compared with enema for disimpaction in children with functional constipation. The risk of bias was assessed using the Cochrane risk of bias tool.

Results: Two RCTs, involving 170 children aged 1 to 17 years, met the inclusion criteria. The studies were generally low in methodological quality. Compared with the enema group, the PEG 3350 group had significantly reduced chance for treatment success, but the difference was of a borderline statistical significance ( $R R=0.83,95 \% \mathrm{Cl}: 0.7-0.99)$. The use of PEG was also more likely to increase defecation frequency, but increased the risk of watery stools and increased faecal incontinence. Other outcomes, in general, were similar in both groups.

Conclusions: Current evidence does not allow us to conclude which intervention is more effective for treating RFI in children with functional constipation. These results should be interpreted with caution due to the limited number of trials and the low quality of reporting in these trials, high or unknown risk of bias, and sparse data. Further high-quality, adequately powered RCTs are needed to determine the optimal management.
\end{abstract}

\section{Introduction}

Constipation is one of the most common digestive complaints in children. It accounts for approximately $3 \%$ of general paediatric visits and $25-30 \%$ of referrals to paediatric gastroenterologists. In $95 \%$ of patients it is of functional origin and usually starts after an episode of painful bowel movements, which leads to withholding behaviour with a cycle of fear and further stool retention. Consequently, a large faecal mass accumulates in the rectum, which is the reason for many complaints such as abdominal pain, excessive flatulence, nausea or vomiting, mood changes with irritability, and most commonly painful bowel movements [1-4]. Severe longstanding constipation is a serious psychological, social, and educational burden for the child's development and a reason for distress for the whole family.

The treatment goal in children with constipation includes rectal faecal disimpaction and then maintaining a regular bowel movement routine. Current guidelines developed by scientific organisations such as the European Society for Paediatric Gastroenterology, Hepatology, and Nutrition (ESPGHAN) and the North American Society for Paediatric Gastroenterology, Hepatology, and Nutrition (NASPGHAN) emphasise that until disimpaction has been achieved, maintenance therapy is not effective [5].

Rectal faecal disimpaction can be accomplished with enemas (with sodium phosphate, saline, or mineral oil solutions) or oral agents such as polyethylene 
glycol (PEG) with or without electrolytes. However, there is uncertainty which regimen is optimal for disimpaction.

\section{Aim}

The aim of this systematic review was to compare the effectiveness and tolerability of PEG versus enema in the management of rectal faecal impaction (RFI) in children with constipation.

\section{Material and methods}

\section{Criteria for considering studies for this review}

Only randomised controlled trials (RCTs) carried out in children (up to 18 years old) with functional constipation and RFI were included. The RFI had to be diagnosed by the physician performing abdominal and/or rectal examination. Trials in subjects with an organic cause for constipation or with a history of colorectal surgery were excluded. The intervention had to be the administration of enema compared with oral administration of PEG. The primary outcome measure was the treatment success as defined by the investigators. The secondary outcome measures were frequency of stool defecations, tolerability of the medication, and adverse events. Other outcomes were included if relevant to the current review.

\section{Search methods for identification of studies}

The Cochrane Central Register of Controlled Trials (CENTRAL, the Cochrane Library), MEDLINE, and EMBASE were systematically searched up to July 2014. The reference lists of identified studies and key review articles were also searched for all studies that assessed the effects of disimpaction treatment in children. Two registries for clinical trials (www.clinicaltrials.gov; www. clinicaltrialsregister.eu) were screened to identify published and ongoing studies. No language restrictions were imposed. Letters to the editor, abstracts, and proceedings from scientific meetings were excluded a priori from the analysis.

The search strategy included the use of a validated filter for identifying RCTs, which was combined with a topic-specific strategy using the following PubMed MeSH terms: (polyethylene glycol OR PEG OR macrogol* or enema* or disimpaction) and (fecal impaction or faecal impaction or functional constipation). Three of the reviewers (PD, $\mathrm{AH}, \mathrm{KW}$ ) searched the databases independently and screened bibliographies of retrieved studies and recent review articles.

\section{Selection of studies}

We excluded studies if the title and abstract were not relevant; however, we retained papers for all potentially relevant studies if the abstract contained insufficient information to warrant exclusion. All areas of disagreement were discussed until a consensus was achieved.

\section{Data extraction and management}

Data from each study were extracted by all of the reviewers using standardised data extraction forms. After extraction, all data were compared in order to minimise the possibility of errors.

\section{Assessment of risk of bias in included studies}

The reviewers, independently, but without being blinded to the authors or journal, assessed the risk of bias in the studies that met the inclusion criteria. We used the Cochrane Collaboration tool for assessing the risk of bias. The following criteria were used: type of randomisation method (to assess the risk of selection bias), allocation concealment (selection bias), blinding of participants and personnel (performance bias), blinding of outcome assessment (detection bias), and presence of intention-to-treat analysis (attrition bias). A low risk of bias was indicated by an answer of "yes" and a high risk of bias by an answer of "no". Inconsistencies were resolved by discussion among the three authors responsible for searching the databases.

\section{Measures of treatment effect and assessment of heterogeneity}

The dichotomous outcomes, the results for individual studies, and pooled statistics are reported as the risk ratio $(\mathrm{RR})$ between the experimental and control groups with $95 \%$ confidence intervals $(\mathrm{CI})$. The mean difference (MD) between the treatment and control groups was selected to represent the difference in continuous outcomes (with $95 \% \mathrm{Cl}$ ). In this paper, we only reported data at the end of both interventions (even if one lasted for less time). Interim data, even if reported by the authors of the original trials, were not presented. Statistical heterogeneity was quantified by $\chi^{2}$ and $R^{2}$, which can be interpreted as the percentage of the total variation between studies that is attributable to heterogeneity rather than to chance. A value of $0 \%$ indicates no observed heterogeneity, and larger values show increasing heterogeneity. As no substantial heterogeneity (over 50\%) was found, the analyses were based on the fixed-effects model. 
The data was analysed using the computer program Review Manager [Computer program. version 5.2. Copenhagen: The Nordic Cochrane Centre, The Cochrane Collaboration, 2012].

\section{Results}

\section{Included studies}

The literature search yielded 97articles, of which 6 were reviewed in full text (Figure 1). Of these studies, only 2 RCTs, both published in English, met the inclusion criteria $[6,7]$. Characteristics of the included trials are summarised in Table I. Characteristics of the excluded trials are presented in Table II. The 2 selected trials randomised a total of 170 children aged 1 to 17 years. In both trials the sample size was appropriately determined, but otherwise they had a number of methodological limitations (Table I). The major limitations were unclear randomisation (one trial), unclear allocation concealment (one trial), no blinding (both trials), and no intention to treat analysis (both trials).

The first RCT, conducted in the tertiary hospital in Amsterdam, the Netherlands, included 90 children (aged $4-16$ years, mean age $7.5 \pm 2.8$ years) with functional constipation and RFI to receive either PEG 3350 with electrolytes $(1.5 \mathrm{~g} / \mathrm{kg} /$ day) or enemas (dioctyl sulfosuccinate sodium). Both interventions were administered once daily for 6 days. Outcomes were measured before and after disimpaction. The authors concluded that both interventions were equally effective in treating RFI in children.

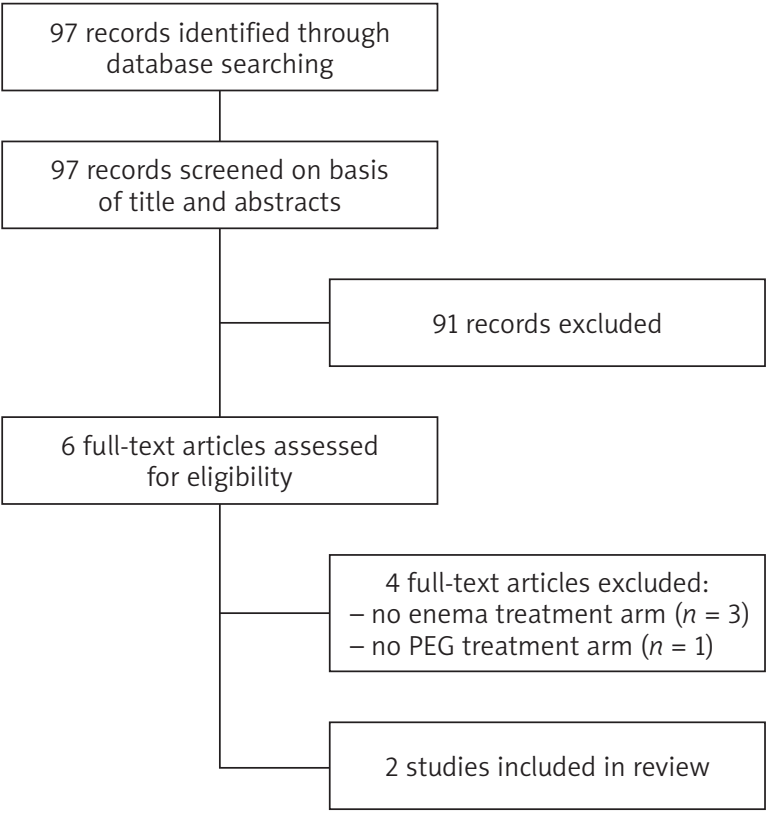

Figure 1. Study flow diagram

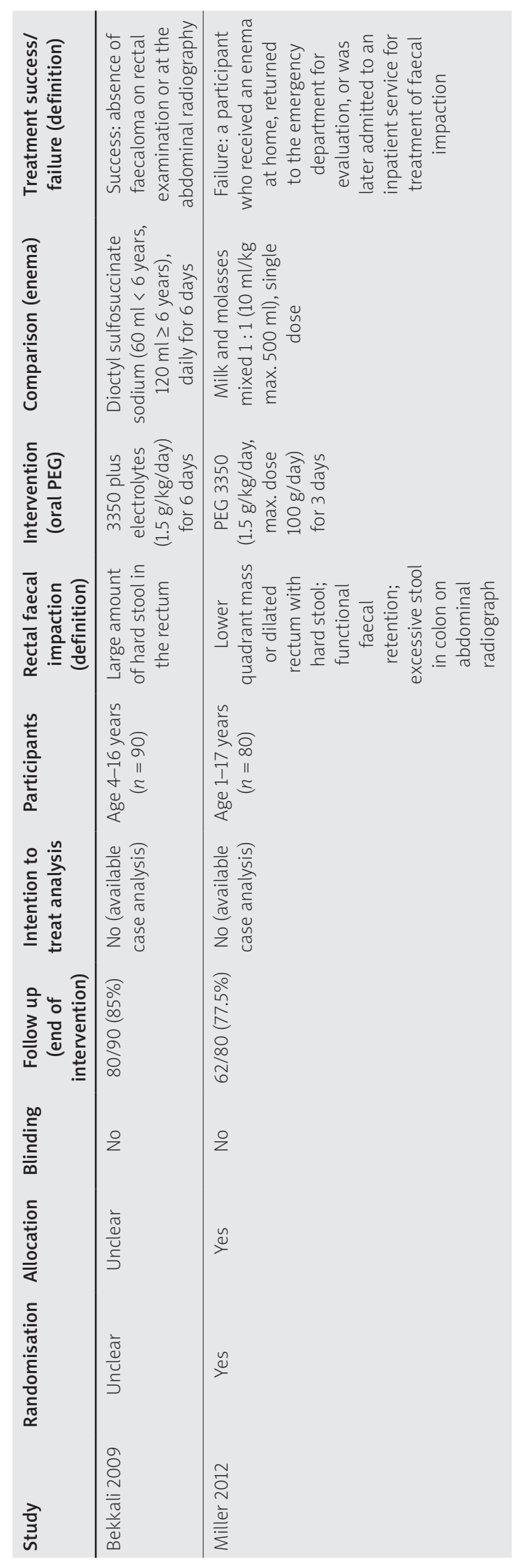


Table II. Defecation frequency

\begin{tabular}{lccccc} 
Ref. & \multicolumn{1}{c}{ Variable } & PEG group & Enema group & Value of $p$ & MD (95\% Cl)* \\
\hline Bekkali & Times per week, mean (SD) & $8.8(8.5)$ & $5.8(3.6)$ & 0.64 & $3.0(0.28-5.72)$ \\
\hline Miller & Day 3 (end of the intervention) (mean) & 4.2 & 2.7 & $<0.05$ & N/A
\end{tabular}

${ }^{*}$ Calculated by the authors of the review.

An RCT conducted in the USA compared the efficacy of the faecal impaction using either PEG 3350 (it was unclear whether it contained electrolytes or not) for 3 days or single enema (milk and molasses) in an out-patient setting. The study involved 79 children aged 1 to 17 years with functional constipation. Children were followed-up by telephone on day 1, 3, and 5. At day 3 (end of intervention), no significant differences were found.

\section{Effects of interventions}

\section{Primary outcome - treatment success}

Both identified RCTs reported similar chance of treatment success, although defined differently. A meta-analysis of these 2 RCTs showed that, compared with the enema group, the PEG group had a significantly reduced rate of treatment success ( $R R=0.83,95 \% \mathrm{Cl}$ : 0.7-0.99) (Figure 1). No significant heterogeneity was found $\left(R^{2}=0 \%\right)$.

\section{Secondary outcomes}

Both trials reported on stool frequency. Based on the calculations made by the reviewers, both found a significant increase in the number of stools in the PEG group compared with the enema group (Table II). However, in the original papers only the authors of one RCT [7] reported that the difference was statistically significant.

Figures 2-4 present the summary of other dichotomous and continuous outcomes, respectively. Com-

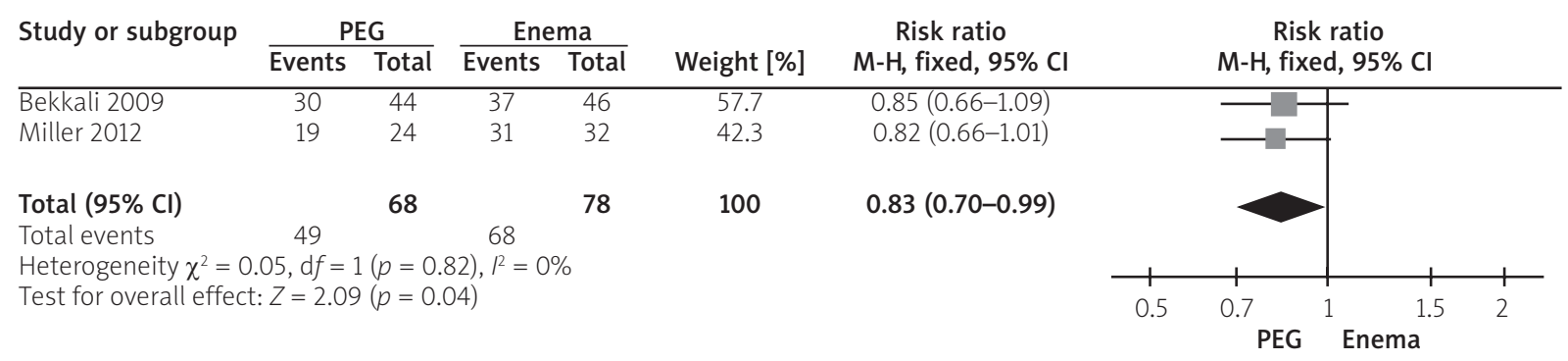

Figure 2. Effect of oral PEG versus PEG enema for rectal faecal disimpaction - treatment success

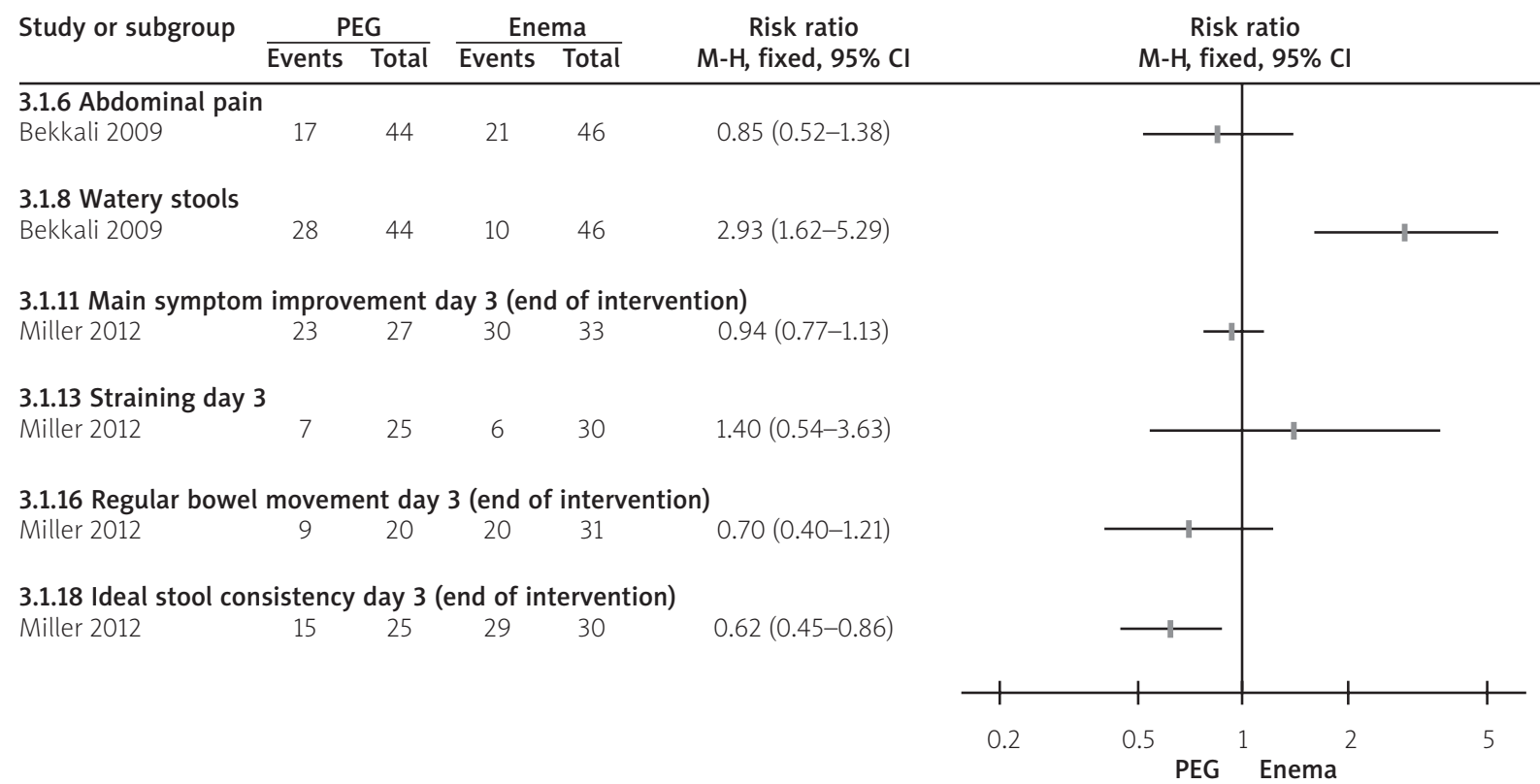

Figure 3. Effect of oral PEG versus PEG enema for rectal faecal disimpaction (dichotomous data) 


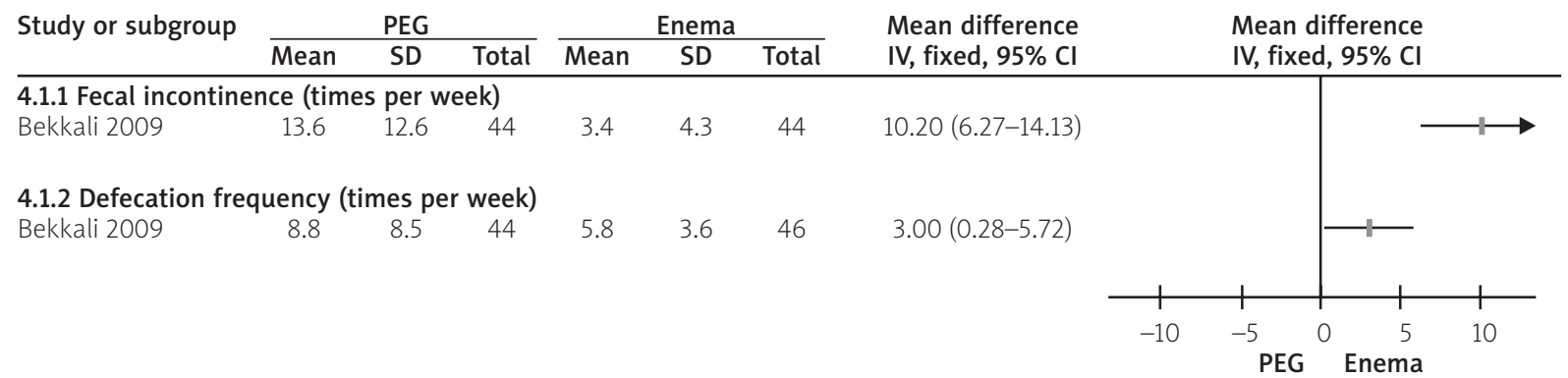

Figure 4. Effect of oral PEG versus PEG enema for rectal faecal disimpaction (continuous data)

pared with the enema group, the PEG group had an increased risk of watery stools ( $R R=2.93,95 \% \mathrm{Cl}$ : 1.62-5.29), increased number of faecal inconsistency episodes per week (MD =10.2, 95\% Cl: 6.27-14.13), and reduced chance for ideal stool consistency on day 3 $(\mathrm{RR}=0.62,95 \% \mathrm{Cl}: 0.45-0.86)$. No significant differences were found with regard to all remaining outcomes.

\section{Discussion}

\section{Summary of evidence}

The aim of this systematic review was to resolve the uncertainty about which regimen is optimal for the treatment of childhood faecal disimpaction. Two RCTs comparing PEG with enema were included. Individually, each RCT reported similar effects of both regimens at the end of intervention. However, in a meta-analysis of these two studies, PEG was found to be significantly less effective than enema for treatment success, although the difference was of a borderline significance. The use of PEG was also more likely to increase defecation frequency, but increased the risk of watery stools and increased faecal incontinence. Other outcomes, in general, were similar in both groups. The findings, whether positive or negative, should be interpreted with caution due to the low quality of the reporting in these studies, a high or unknown risk of bias associated with the trials in this pooled analysis, and sparse data.

\section{Strengths and limitations}

The strength of this review is the use of a rigorous systematic review methodology. Our search included three major databases, with no language restrictions. However, no attempts were made to identify unpublished trials. Consequently, we cannot fully exclude the possibility of publication bias, which is a significant threat to the validity of any systematic review. Only a limited number of trials were available for this review. While both trials included the sample size calculation, the methodological quality of the trials varied. Potential limitations included unclear sequence generation, unclear allocation concealment, no blinding, and no intention-to-treat analysis. Noteworthy are the differ- ences in the duration of interventions and the dose of enema. There was also no agreed-upon definition of the treatment success as well as other outcomes.

\section{Conclusions}

Current evidence does not allow us to conclude which intervention is more effective for treating RFI in children with functional constipation. The findings of this review should be interpreted with caution due to the low quality of reporting in these trials, high or unknown risk of bias, and sparse data. Further high-quality, adequately powered RCTs are needed to determine the optimal management.

\section{Conflict of interest}

The authors declare no conflict of interest.

\section{References}

1. van der Berg MM, Benninga MA, Di Lorenzo C. Epidemiology of childhood constipation: a systematic review. Am J Gastroenterol 2006; 101: 2401-9.

2. Benninga MA, Voskuijl WP, Taminiau JA. Childhood constipation: is there new light in the tunnel? J Pediatr Gastroenterol Nutr 2004; 39: 448-64.

3. Gibas-Dorna M, Piątek J. Functional constipation in children evaluation and management. Prz Gastroenterol 2014; 9: 194-9.

4. van Ginkel R, Reitsma JB, Büller HA, et al. Chilhood constipation: longitudinal follow-up beyond puberty. Gastroenterology 2003; 125: 357-63.

5. Tabbers MM, Dilorenzo C, Berger MY, et al. Evaluation and treatment of functional constipation in infants and children: evidence-based recommendations from ESPGHAN and NASPGHAN. J Pediatr Gastroenterol Nutr 2014; 58: 265-81.

6. Bekkali NL, van den Berg MM, Dijkgraaf MG, et al. Rectal fecal impaction treatment in childhood constipation: enemas versus high doses oral PEG. Pediatrics 2009; 124: e1108-5.

7. Miller MK, Dowd MD, Friesen CA, et al. A randomized trial of enema versus polyethylene glycol 3350 for fecal disimpaction in children presenting to an emergency department. Pediatr Emerg Care 2012; 28: 115-9.

Received: 5.12 .2014

Accepted: 21.01.2015 